\title{
A FUNCTION WHICH IS WEAKLY OF BOUNDED VARIATION BUT DOES NOT HAVE THE GOWURIN $\omega$-PROPERTY
}

\section{H. TUCKER}

Suppose each of $X$ and $Y$ is a linear normed space, $B[X, Y]$ is the space of bounded linear transformations from $X$ in to $Y$ and $B^{+}[X, Y]$ is the weak sequential extension [2] of $B[X, Y]$. It is shown in [2] that elements of $B^{+}[X, Y]$ can be considered as elements of $B\left[X, Y^{+}\right]$. A function $K$ from $[0,1]$ into $B^{+}[X, Y]$ is said to satisfy the Gowurin $\omega$-property [1] provided there exists an $M>0$ such that if $0=t_{0} \leqq t_{1} \leqq \cdots \leqq t_{n}=1$ is a subdivision of $[0,1]$ and $x_{0}, x_{1}, \cdots$, $x_{n-1}$ are any $n$ points in $X$, then

$$
\left\|\sum_{i=0}^{n-1}\left[K\left(t_{i+1}\right)-K\left(t_{i}\right)\right] \cdot x_{i}\right\| Y^{+} \leqq M \max \left\|x_{i}\right\|_{X} .
$$

Some writers call this property bounded semivariation.

In the lemma of [2], it is shown that if $K$ has the $\omega$-property, then if $F$ is in $B^{*}\left[X, Y^{+}\right]$, then $F K$ is of bounded variation on $[0,1]$ and $V_{0}^{1} F K \leqq\|F\| W_{0}^{1} K$, where $W_{0}^{1} K$ is the inf of all numbers $M$ above. We shall refer to this property as being weakly of bounded variation. This lemma was used to obtain a uniqueness theorem (Theorem 2) for the generating functions of bounded linear transformations from $C(X)=C$, the continuous functions from $[0,1]$ into $X$, into $Y$. It was also observed that a better form of the uniqueness theorem could be stated if one could prove the converse of the above lemma. A partial converse was obtained (Theorem 3), namely that if $K$ is weakly of bounded variation, then it satisfies a real $\omega$-property, that is, it satisfies the $\omega$-property where the points $x_{i}$ are replaced by real numbers $r_{i}$. This gives the improved version of Theorem 2 in the case that $X$ is the real line.

The purpose of this note is to give an example of a function which is weakly of bounded variation but does not have the $\omega$-property. It will follow that Theorem 2 can not in general be improved.

EXAmple. We shall denote by $C_{0}$ the space of all real functions $f$ on $[0,1]$ such that given $\epsilon>0$, the set of all $s$ in $[0,1]$ such that $|f(s)| \geqq \epsilon$ is finite. This space is endowed with the sup norm. Let $H$ denote the space of all real functions $f$ on $[0,1]$ such that $\sum f^{2}\left(s_{i}\right)<\infty$

Received by the editors December 8, 1966. 
where $\left\{s_{i}\right\}$ is the set of all points $s$ such that $f(s) \neq 0$ and we define $\|f\|_{H}=\left[\sum f^{2}\left(s_{i}\right)\right]^{1 / 2}$.

Given $f$ in $C_{0}$ and $g$ in $H$ we define $(f g)(s)=f(s) g(s)$ and note that $f g$ is in both $H$ and $C_{0}$ and $f$ may be considered an element of $B[H, H]$. That this map is continuous follows at once from the fact that $\|f g\|_{H}$ $\leqq\|f\|_{C_{0}}\|g\|_{H}$ and indeed $\|f\|_{B[H, H]}=\|f\|_{C_{0}}$. Hence we may consider $C_{0}$ as a subspace of $B[H, H]$.

For this example we shall take $X=Y=H$ and we define $K$ from $[0,1]$ into $C_{0} \subset B[H, H]$ as follows: $K(t)=\chi_{t}(s)$ where $\chi_{t}(s)=0$ if $t \neq s, \chi_{t}(s)=1$ if $t=s$.

We shall first show that $K$ does not have the $\omega$-property. Suppose $\sigma$ is a partition $0=t_{0} \leqq t_{1} \leqq \cdots \leqq t_{n}=1$ and consider

$$
\begin{aligned}
\| \sum_{i=0}^{n-1}\left(\left[K\left(t_{i+1}\right)-\right.\right. & \left.\left.K\left(t_{i}\right)\right] x_{i}\right)(s) \|_{H} \\
= & {\left[\sum_{j=0}^{\infty}\left[\sum_{i=0}^{n-1}\left(\left[K\left(t_{i+1}, s_{j}\right)-K\left(t_{i}, s_{j}\right)\right] x_{i}\right)\left(s_{j}\right)\right]^{2}\right]^{1 / 2} }
\end{aligned}
$$

where $\left\{s_{j}\right\}$ is the collection of all nonzero points for the functions $x_{0}, x_{1}, \cdots, x_{n-1}$ in $H$. Now for this partition $\sigma$, we choose $x_{i}(s)$ $=K\left(t_{i}, s\right)$. We then have that $\left\|x_{i}\right\|_{H}=1$ and

$$
\begin{aligned}
\mid \sum_{i=0}^{n-1}\left[K\left(t_{i+1}, s_{j}\right)\right. & \left.-K\left(t_{i}, s_{j}\right)\right]\left.x_{i}\left(s_{j}\right)\right|^{2} \\
= & \left|\sum_{i=0}^{n-1} K\left(t_{i+1}, s_{j}\right) K\left(t_{i}, s_{j}\right)-K^{2}\left(t_{i}, s_{j}\right)\right|^{2}=1
\end{aligned}
$$

for each $j=0,1, \cdots, n-1$ and is zero otherwise; thus, we have that

$$
\left\|\sum_{i=0}^{n-1}\left[K\left(t_{i+1}\right)-K\left(t_{i}\right)\right] x_{i}\right\|_{H}=n^{1 / 2}
$$

which is unbounded. Therefore, $K$ does not have the $\omega$-property.

To see that $K$ is weakly of bounded variation we first note that any $F$ in $B^{*}[H, H]$ when restricted to $C_{0} \subset B[H, H]$ is an element of $C_{0}^{*}$ and thus we need only consider such functionals. It is an easy exercise to show that each $F$ in $C_{0}^{*}$ when applied to an $f$ in $C_{0}$ can be represented by $F(f)=\sum f\left(s_{i}\right) F\left(K\left(s_{i}\right)\right)<\infty$ where the sum is taken over all those $s$ in $[0,1]$ such that $f(s) \neq 0$. Since this sum must be finite for all $f$ in $C_{0}$, it follows that there exists at most countably many points $s_{i}$ in $[0,1]$ such that $F\left[K\left(s_{i}\right)\right] \neq 0$ and that for such points $\sum\left|F\left[K\left(s_{i}\right)\right]\right|<\infty$. Thus $F K$ is of bounded variation for each $F$. 
If for a given pair of spaces $X$ and $Y$, we denote those functions $K$ which are weakly of bounded variation by $w b v$, those which have the Gowurin $\omega$-property by $G$ and those which are of bounded variation by $B V$, then we may summarize our results as follows: $w b v G \supset B V$.

The first inclusion follows from the lemma of [2] and the second follows trivially from the triangle inequality. Furthermore, in general the inclusions are proper, the first by the above example and the second by the example in the introduction of [2]. (There $X$ is the real line.) However, if $X$ is $Y$ is the real line, then $G$ is $B V$. By Theorem 3 [2] if $X$ is the real line, then $w b v$ is $G$. It would be of interest to know a characterization of those spaces $X$ such that $w b v$ is $G$ for all $Y$.

Theorem 2 of [2] is stated as follows: In order that two functions $K_{1}$ and $K_{2}$ should generate the same continuous linear transformation $T$ in $B[C, Y]$ it is necessary and sufficient that

(i) each of $K_{1}$ and $K_{2}$ generate such a $T$ and

(ii) there exists a point $d$ in $B^{+}[X, Y]$ such that $K_{1}(0)-K_{2}(0)$ $=K_{1}(1)-K_{2}(1)=d$ and for each $F$ in $B^{*}\left[X, Y^{+}\right], F\left[K_{1}(t)-K_{2}(t)\right]$ $=F(d)$ except possibly over a countable set $E(F)$ of points and that for the points $e$ in $E(F), \sum_{E(F)}\left|F\left[K_{1}(e)-K_{2}(e)\right]\right|$ converges.

The proposed improvement involved replacing the word "each" in (i) by the word "one." We indicated in [2] that this can be done in case $X$ is the real line. Our present example shows that this can not always be done. To see this one need only consider $K^{\prime}(t)=K(t)$ if $0<t<1, K^{\prime}(1)=K^{\prime}(0)=0$, and then choose any $K_{1}$ (with the $\omega$ property) in our present set up which does generate such a $T$ and add $K^{\prime}$ to it. The resulting function does not have the $\omega$-property, hence does not generate a $T$, and the difference of the two, namely $K^{\prime}$ itself, does satisfy (ii).

\section{BIBLIOGRAPHY}

1. Mark Gowurin, Über die Stieltjessche Integration abstrakter Functionen, Fund. Math. 27 (1936), 254-268.

2. D. H. Tucker, A representation theorem for a continuous linear transformation on a space of continuous functions, Proc. Amer. Math. Soc. 16 (1965), 946-953.

UNIVERSITÄT MARBURG AND

UNIVERSITY OF UTAH 